\title{
An evaluation of voice conversion with neural network spectral mapping models and WaveNet vocoder
}

\author{
PATRICK LUMBAN TOBING, ${ }^{1}$ (1) YI-CHIAO WU, ${ }^{1}$ TOMOKI HAYASHI, ${ }^{1}$ KAZUHIRO KOBAYASHI ${ }^{2}$ AND \\ TOMOKI TODA ${ }^{2}$
}

\begin{abstract}
This paper presents an evaluation of parallel voice conversion (VC) with neural network (NN)-based statistical models for spectral mapping and waveform generation. The NN-based architectures for spectral mapping include deep $N N(D N N)$, deep mixture density network (DMDN), and recurrent $N N(R N N)$ models. WaveNet $(W N)$ vocoder is employed as a high-quality NN-based waveform generation. In VC, though, owing to the oversmoothed characteristics of estimated speech parameters, quality degradation still occurs. To address this problem, we utilize post-conversion for the converted features based on direct waveform modifferential and global variance postfilter. To preserve the consistency with the post-conversion, we further propose a spectrum differential loss for the spectral modeling. The experimental results demonstrate that: (1) the RNN-based spectral modeling achieves higher accuracy with a faster convergence rate and better generalization compared to the DNN-/DMDNbased models; (2) the RNN-based spectral modeling is also capable of producing less oversmoothed spectral trajectory; (3) the use of proposed spectrum differential loss improves the performance in the same-gender conversions; and (4) the proposed post-conversion on converted features for the WN vocoder in VC yields the best performance in both naturalness and speaker similarity compared to the conventional use of $W N$ vocoder.
\end{abstract}

Keywords: Voice conversion, Neural network, Spectral mapping, WaveNet vocoder, Oversmoothed parameters

Received 15 July 2020; Revised 22 October 2020

\section{INTRODUCTION}

Through a voice conversion (VC) [1] system, voice characteristics of a source speaker can be transformed into those of the desired target speaker while still preserving the linguistic information. VC has been applied to various speech applications, such as for generation of speech databases with various voice characteristics $[2,3]$, singing voice conversion $(\mathrm{VC})[4,5]$, the recovery of impaired speech signals $[6-8]$, expressive speech synthesis $[9,10]$, body-conducted speech processing $[11,12]$, and speech modification with articulatory control [13]. Furthermore, recent developments of anti-spoofing countermeasure systems $[14,15]$ have also employed VC systems for part of the spoofing data. Therefore, considering the benefits of VC, it is certainly worthwhile conducting a thorough study of the development of a high-quality VC system.

\footnotetext{
${ }^{1}$ Graduate School of Information Science, Nagoya University, Nagoya, Aichi 4648601, Japan

${ }^{2}$ Information Technology Center, Nagoya University, Nagoya, Aichi 464-86o1, Japan

Corresponding author:

Patrick Lumban Tobing

Email: patrick.lumbantobing@g.sp.m.is.nagoya-u.ac.jp
}

To perform VC, in general, two high-level features of a speech signal are used in the conversion, namely the voicetimbre and prosody characteristics. To convert the voicetimbre, one convenient way to do it is by transforming a compact representation of the vocal tract spectrum, such as mel-cepstrum features [16], through the use of a data-driven statistical mapping. Although there are several data-driven mapping for prosody transformation [17-19], in this work, we focus on the use of data-driven mappings for the conversion of spectral parameters. Finally, these transformed speech features are used to generate the converted speech, for example by using a vocoder-based waveform generation [20-22], or possibly by using a data-driven statistical waveform generation $[23,24]$.

Indeed, in recent years, the development of data-driven VC systems have been rapidly proceeding, such as VC with a codebook-based method [2], with frequency-warping methods [25, 26], with exemplar-based mappings [27, 28], with statistical methods using Gaussian Mixture Model (GMM)-based approaches $[3,22,29]$, and with neuralnetwork (NN)-based models [30-35]. In this work, considering the potential of NN-based methods, we focus on its use to perform the spectral conversion in VC, particularly with recurrent neural network (RNN) $[36,37]$, which 
can capture the long-term context dependencies. Note that different from recent works on the use of non-parallel (unpaired) data, such as with restricted Boltzmann machine [33], variational autoencoder [34], or generative adversarial network [35], in this work, we focus on the development of NN-based VC with parallel data. This is because in many cases it is still viable to collect a small amount of parallel data, i.e. where the source and target speakers utter the same set of sentences. Furthermore, established RNNbased modeling in parallel VC can be adapted for improving non-parallel methods.

As has been mentioned, in VC, to generate a converted speech waveform, a vocoder-based framework can be deployed, such as the mixed-excitation-based vocoders (STRAIGHT [21], WORLD [38]). Other highquality vocoders have also been developed, such as glottalexcitation-based vocoders $[39,40]$ and sinusoidal vocoders $[41,42]$. A comparison of these vocoders on analysis-synthesis cases has also been made [40, 43]. However, in cases where the speech features are estimated from a statistical model, such as from a text-to-speech (TTS) system, the performance of these vocoders is highly degraded [40, 42]. The degradation in TTS, and possibly VC, applications are caused by the non-ideal conditions of the speech features estimated from a statistical model, such as oversmoothed condition of the spectral trajectory, where its variance is highly reduced due to the mean-based optimization [22]. To overcome this problem, in this work, we investigate the use of the data-driven waveform generation method, which offers more potential in compensatory capability than the rule-based conventional vocoder.

Recently, a data-driven NN-based waveform generation method, using a deep autoregressive (AR) convolutional neural network (CNN) called WaveNet (WN) [23] has been proposed. WN models the waveform samples based on their previous samples using a stack of dilated CNNs to efficiently increase the number of receptive fields. In [44-46], auxiliary conditioning speech parameters, such as spectral and excitation features, are used to develop a state-of-theart WN vocoder, which could generate meaningful speech waveforms with natural quality. However, as in a conventional vocoder, in VC [47], WN still suffers from quality degradation due to the use of oversmoothed speech features, which introduces mismatches to the natural speech features used in training the model. To alleviate this problem, in this work, we presume that postprocessing methods $[5,22,48]$ will be more helpful in a WN vocoder, compared to the conventional vocoder, thanks to its data-driven characteristic for statistical compensation.

In this paper, to develop a better VC system, we employ the use of NN architectures for both spectral conversion modeling and waveform generation modeling. First, we perform an investigation on the use of RNN-based spectral conversion models compared to the conventional deep neural network (DNN) and deep mixture density network (DMDN) models [24] in a limited data condition. We show that our proposed RNN-based architecture could yield better performance, and it naturally can capture long-term context dependencies in training and mapping stages. Furthermore, to improve the condition of the generated spectral trajectory, we also propose to use spectrum differential loss for the RNN-based model training, which is based on a synthesis/postfilter approach using direct waveform modification with spectrum differential (DiffVC) [5]

Lastly, to improve the statistical waveform modeling with a $\mathrm{WN}$ vocoder in $\mathrm{VC}$, we propose several postprocessing methods that alleviate the possible mismatches and oversmoothness of the estimated speech features. These postprocessing methods are based on global variance $(\mathrm{GV})$ postfilter [22] and the DiffVC method [5]. In the future, it would be better to avoid the use of such postprocessing techniques and directly address the feature mismatches by utilizing the data-driven traits of a statistical waveform model. Indeed, in both TTS [49] and VC [50], such a concept has been applied, where text/linguistic features are used, which may not be available in every practical situation. In this work, as the first step toward the improvement of a more flexible text-independent VC system, we perform investigations on the capability of statistical waveform modeling with postprocessing techniques.

To recap, in this paper, our contributions are twofold: to develop an RNN-based spectral conversion model that is better than the conventional DNN architectures, and to investigate the effects of several postprocessing methods for improving the use of $\mathrm{WN}$ vocoder in VC. In the experimental evaluation, it has been demonstrated that: (1) the proposed RNN-based spectral modeling architecture achieved better performance than the conventional DNN/DMDN architectures even with limited training data; (2) the proposed spectrum differential loss in RNN-based modeling further improves the naturalness of converted speech in same-gender conversions; and (3) the proposed post-conversion processing yields the best naturalness and conversion accuracy of converted speech compared to the conventional use of WN vocoder. Henceforth, the main motivation of this work is to better understand the effectiveness of using NN-based feature modelings, such as for spectral mapping, and waveform generation, i.e. neural vocoder, through careful evaluations and investigations toward further improvements of these core techniques.

The remainder of this paper is organized as follows. In Section II, a brief overview of the proposed systems and their correlation with the previous work are described. In Section III, the proposed NN-based architectures for the spectral mapping models are elaborated. In Section IV, the WN vocoder used as the NN-based waveform model is explained, as well as the proposed method for alleviating the quality degradation in VC. In Section V, objective and subjective experimental evaluations are presented. Finally, the conclusion of this paper is given in Section VI.

\section{COMPARISON TO PREVIDUS W $口 R K$}

In this work, we describe our method for a VC system with the use of NN-based statistical models for both spectral 

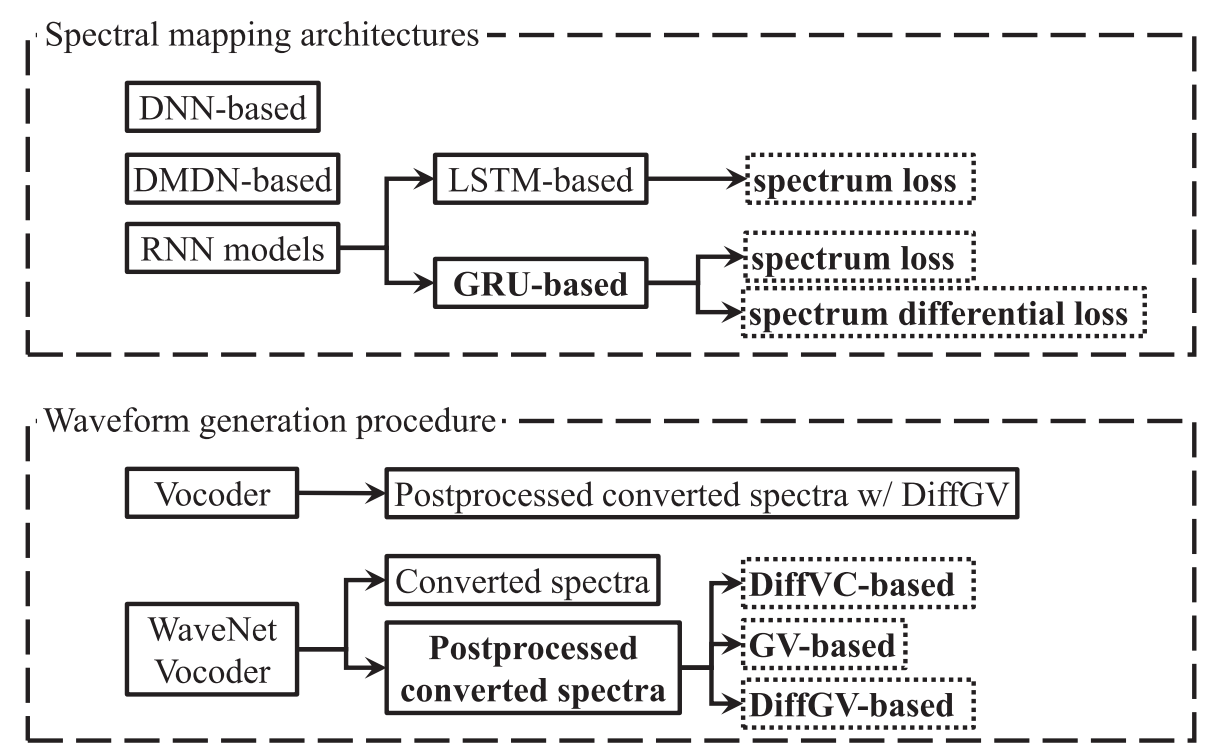

Fig. 1. Diagram of the overall flow and contribution of the proposed work. Bold denotes proposed methods and dashed boxes denote proposed experimental comparisons. Arrow denotes a particular implementation detail of its parent module.

mapping and waveform generation. This idea was incorporated in our previous VC system [24], which participated in the VCC 2018 [51]. The previous system [24], which was ranked second in the challenge, used a DMDN-based model [52] for the spectral mapping and a WN vocoder to generate the converted speech. To alleviate the degradation problem in $\mathrm{WN}$, a post-conversion processing based on the DiffVC and GV (DiffGV) was used to obtain refined auxiliary speech features for the WN vocoder. However, there still exist several limitations on our previous work, which include (1) the need of using a separate module in the DMDN-based model to incorporate time-dependency in spectral trajectory generation, i.e. maximum likelihood parameter generation (MLPG) [22, 53]; (2) no empirical observations that prove the DiffGV post-conversion processing is better than either solely DiffVC- or GV-based; and (3) a possibility of better spectral modeling with spectrum differential loss to preserve the consistency with DiffGV method, which uses spectrum differential features in its process.

To build on our previous work, first, we propose to improve the spectral mapping modeling, as illustrated in the upper diagram of Fig. 1, as follows: (1) by using RNN-based architecture, which can naturally capture long-term context dependencies, and (2) by using spectrum differential loss in parameter optimization to preserve the consistency with the DiffGV method, which may improve the condition of generated spectral trajectory. To clearly describe these methods, we first elaborate on the DNN- and DMDN-based spectral mapping models $[24,52]$. We also describe the RNN-based architectures for spectral mapping with long-short term memory (LSTM) [36] or with a gated recurrent unit (GRU) [37]. Finally, these spectral mapping models are objectively evaluated in a limited training data condition, where it has been demonstrated that the GRU-based spectral modeling yields better performance than the others. Note that we focus on comparing the LSTM- and GRU-based spectral modeling in a basic VC task to confirm the effectiveness of a more compact GRU architecture, which is more beneficial for real-world applications.

Secondly, we propose to investigate the effect of several post-conversion processing methods, i.e. with DiffVC-, with GV-, or with DiffGV-based post-conversions, for the $\mathrm{WN}$ vocoder in VC. We also compare these methods with the conventional vocoder framework that uses DiffGVbased post-processed speech features, as illustrated by the lower diagram of Fig. 1. In the experimental evaluation, it has been demonstrated that the DiffGV-based postconversion with $\mathrm{WN}$ vocoder yields superior performance compared to the others. Further, coupled with the use of the proposed spectrum differential loss in the spectral mapping model, the proposed method yields higher performance in the same-gender conversions.

\section{SPECTRAL CONVERSION MODELS WITH NN-BASED ARCHITECTURES}

This section describes the NN-based spectral mapping models used for the VC framework in this paper. These include the DNN and DMDN [52], which are optimized according to the conditional probability density function (PDF) of the target spectral features, given the input source spectral features. Compare to the straightforward DNN architecture, the DMDN has the advantage of being capable of modeling a more complex distribution of the target features, as well as to model not only their means but also their variances. In the DNN and DMDN, a separate MLPG module $[22,53]$ is used after the conversion network to generate the estimated spectral trajectory. Finally, we also elaborate on the RNN-based spectral mapping models, where, in contrast to the DNN/DMDN models, all components can be optimized during training. 
Let $\quad \boldsymbol{x}_{t}=\left[x_{t}(1), \ldots, x_{t}(d), \ldots, x_{t}(D)\right]^{\top} \quad$ and $y_{t}=\left[y_{t}(1), \ldots, y_{t}(d), \ldots, y_{t}(D)\right]^{\top}$ be the $D$-dimensional spectral feature vectors of the source speaker and target speaker, respectively, at frame $t$. Their $2 \mathrm{D}$ spectral feature vectors are, respectively, denoted as $\boldsymbol{X}_{t}=\left[\boldsymbol{x}_{t}^{\top}, \Delta \boldsymbol{x}_{t}^{\top}\right]^{\top}$ and $\boldsymbol{Y}_{t}=\left[\boldsymbol{y}_{t}^{\top}, \Delta \boldsymbol{y}_{t}^{\top}\right]^{\top}$, where $\Delta \boldsymbol{x}_{t}$ and $\Delta \boldsymbol{y}_{t}$ are the corresponding delta features, which capture the rate of change of values between successive feature frames.

\section{A) Spectral conversion with DNN}

In the DNN, given an input spectral feature vector $\boldsymbol{X}_{t}$ at frame $t$, the conditional PDF of the target spectral feature vector $\boldsymbol{Y}_{t}$ is defined as

$$
P\left(\boldsymbol{Y}_{t} \mid \boldsymbol{X}_{t}, \boldsymbol{\lambda}_{S}\right)=\mathcal{N}\left(\boldsymbol{Y}_{t} ; f_{\lambda_{S}}\left(\boldsymbol{X}_{t}\right), \boldsymbol{D}\right),
$$

where $\mathcal{N}(\cdot ; \mu, \Sigma)$ denotes a Gaussian distribution with a mean vector $\boldsymbol{\mu}$ and a covariance matrix $\Sigma$. $D$ is the diagonal covariance matrix of the target spectral features computed using the training dataset beforehand. The set of parameters of the network model is denoted as $\lambda_{S}$, and $f_{\lambda_{S}}(\cdot)$ is the feedforward function of the network.

In the training phase of the DNN network, to estimate the updated model parameters $\hat{\lambda}_{S}$, backpropagation is performed throughout the network to minimize the loss function as follows:

$$
\hat{\boldsymbol{\lambda}}_{S}=\arg \min _{\lambda_{S}} \frac{1}{T} \sum_{t=1}^{T}\left(\boldsymbol{Y}_{t}-f_{\lambda_{S}}\left(\boldsymbol{X}_{t}\right)\right)^{\top} \boldsymbol{D}^{-1}\left(\boldsymbol{Y}_{t}-f_{\lambda_{S}}\left(\boldsymbol{X}_{t}\right)\right),
$$

where $\top$ denotes the transpose operator and $T$ denotes the total number of time frames.

Then, using the trained DNN network model, a spectral conversion function can be applied to generate the estimated target spectral trajectory $\hat{\boldsymbol{y}}=\left[\hat{\boldsymbol{y}}_{1}^{\top}, \hat{\boldsymbol{y}}_{2}^{\top}, \ldots, \hat{\boldsymbol{y}}_{t}^{\top}, \ldots\right.$, $\left.\hat{\boldsymbol{y}}_{T}^{\top}\right]^{\top}$ by using the MLPG $[22,53]$ procedure as follows:

$$
\hat{\boldsymbol{y}}=\left(\boldsymbol{W}^{\top} \overline{\boldsymbol{D}}^{-1} \boldsymbol{W}\right)^{-1} \boldsymbol{W}^{\top} \overline{\boldsymbol{D}}^{-1} \boldsymbol{M},
$$

where $\quad \boldsymbol{M}=\left[f_{\lambda_{S}}\left(\boldsymbol{X}_{1}\right)^{\top}, f_{\lambda_{S}}\left(\boldsymbol{X}_{2}\right)^{\top}, \ldots, f_{\lambda_{S}}\left(\boldsymbol{X}_{t}\right)^{\top}, \ldots\right.$, $\left.f_{\lambda_{S}}\left(\boldsymbol{X}_{T}\right)^{\top}\right]^{\top}$ is the sequence of network outputs, and $\overline{\boldsymbol{D}}^{-1}$ denotes a sequence of inverted matrices of the diagonal covariance $\boldsymbol{D}$. The transformation matrix is denoted as $\boldsymbol{W}$, which is used to enhance a sequence of spectral feature vectors with its delta feature contexts, i.e. the rate of change of values between successive feature frames.

\section{B) Spectral conversion with DMDN}

For DMDN-based [52] spectral conversion, given an input spectral feature vector $\boldsymbol{X}_{t}$ at frame $t$, the conditional PDF of the target spectral feature vector $\boldsymbol{Y}_{t}$ is defined as a mixture of distributions as

$$
P\left(\boldsymbol{Y}_{t} \mid \boldsymbol{X}_{t}, \lambda\right)=\sum_{m=1}^{M} \alpha_{m, t, \lambda} \mathcal{N}\left(\boldsymbol{Y}_{t} ; \boldsymbol{\mu}_{m, t, \lambda}, \Sigma_{m, t, \lambda}\right)
$$

where the Gaussian distribution is denoted as $\mathcal{N}(\cdot ; \mu, \Sigma)$ with a mean vector $\boldsymbol{\mu}$ and a covariance matrix $\Sigma$. The index of the mixture component is denoted by $m$ and the total number of mixture components is $M$. The set of model parameters is denoted as $\lambda$. The time-varying weight, mean vector, and diagonal covariance matrix of the $m$ th mixture component are, respectively, denoted as $\alpha_{m, t, \lambda}, \boldsymbol{\mu}_{m, t, \lambda}$, and $\Sigma_{m, t, \lambda}$. These time-varying mixture parameters are taken from the network output

$$
\begin{aligned}
f_{\lambda}\left(\boldsymbol{X}_{t}\right)= & {\left[\alpha_{1, t, \lambda}, \ldots, \alpha_{M, t, \lambda}, \boldsymbol{\mu}_{1, t, \lambda}^{\top}, \ldots, \boldsymbol{\mu}_{M, t, \lambda}^{\top},\right.} \\
& \left.\operatorname{diag}\left(\boldsymbol{\Sigma}_{1, t, \lambda}\right)^{\top}, \ldots, \operatorname{diag}\left(\Sigma_{M, t, \lambda}\right)^{\top}\right]^{\top} .
\end{aligned}
$$

To estimate the updated model parameters $\hat{\lambda}$ in the training of a DMDN-based spectral conversion model, backpropagation is performed according to the following negative log-likelihood function:

$$
\begin{aligned}
\hat{\lambda}= & \arg \min _{\lambda} \frac{1}{T} \sum_{t=1}^{T} \sum_{m=1}^{M} D \log 2 \pi \\
& +\frac{1}{2} \log \left|\Sigma_{m, t, \lambda}\right|-\log \alpha_{m, t, \lambda} \\
& -\frac{1}{2}\left(\boldsymbol{Y}_{t}-\boldsymbol{\mu}_{m, t, \lambda}\right)^{\top} \Sigma_{m, t \lambda}^{-1}\left(\boldsymbol{Y}_{t}-\boldsymbol{\mu}_{m, t, \lambda}\right) .
\end{aligned}
$$

Similar to the DNN, in the conversion phase, the estimated target spectral trajectory $\hat{y}$ is generated by using the MLPG $[22,53]$ procedure as follows:

$$
\hat{y}=\left(W^{\top} \overline{\Sigma_{\hat{m}}^{-1}} W\right)^{-1} W^{\top} \overline{\Sigma_{\hat{m}}^{-1}} \mu_{\hat{m}}
$$

where the transformation matrix used to append the spectral feature vector sequence with its delta features is denoted as $\boldsymbol{W}$. The sequence of mixture-dependent time-varying inverted diagonal covariance matrices is denoted as $\overline{\Sigma_{\hat{m}}^{-1}}$ and that of the mean vectors is denoted as $\overline{\boldsymbol{\mu}_{\hat{\boldsymbol{m}}}}$. The suboptimum mixture component sequence, which is denoted as $\hat{\boldsymbol{m}}=\left\{\hat{m}_{1}, \hat{m}_{2}, \ldots, \hat{m}_{t}, \ldots, \hat{m}_{T}\right\}$, used to develop the respective sequences of time-varying mixture parameters is determined as

$$
\hat{\boldsymbol{m}}=\operatorname{argmax}_{m} \prod_{t=1}^{T} \alpha_{m, t, \lambda} \mathcal{N}\left(\boldsymbol{Y}_{t} ; \boldsymbol{\mu}_{m, t, \lambda}, \Sigma_{m, t, \lambda}\right) .
$$

Note that both (3) and (6) generate the estimated $D$-dimensional target feature vector sequence $\hat{y}$, whereas the input to the network is $2 \mathrm{D}$ input feature vector $\boldsymbol{X}_{t}$ at each time $t$. This is because the network actually outputs $2 \mathrm{D}$ feature vector for DNN, i.e. $f_{\lambda_{S}}\left(\boldsymbol{X}_{t}\right)$, or $M$ sets of $2 \mathrm{D}$ mean vector $\boldsymbol{\mu}_{m, t, \lambda}$ and diagonal covariance matrix $\operatorname{diag}\left(\Sigma_{\mathrm{m}, \mathrm{t}, \lambda}\right)$ for DMDN at each time $t$, where they were then processed by the MLPG procedure to generate the spectral trajectory by considering their temporal correlation. This kind of explicit treatment can be alleviated by directly incorporating the use of network architecture with temporal modeling capabilities, such as RNN, which is described in the following section. 


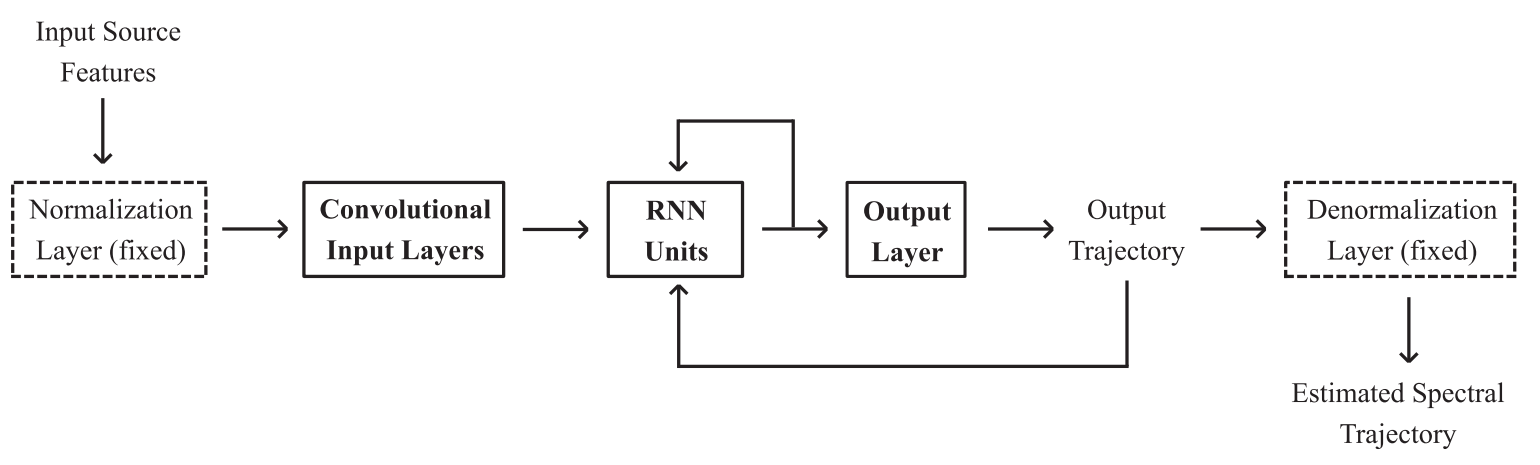

Fig. 2. Spectral mapping model with RNN-based architectures.

\section{C) Spectral conversion with RNN}

It is also important to note that a speech signal, and thus its parametrization, is time-sequence data. Therefore, it would be more reasonable to develop a spectral conversion model that is capable of capturing the dependencies within a sequence of spectral feature vectors, such as by using the RNN architectures. Hence, we propose the use of LSTM [36] and a GRU [37] as the basis RNN units used to capture the long-term context dependencies on the input spectral features. The flow of the RNN-based spectral modeling is shown in Fig. 2. It can be observed that additional convolutional input layers are also used to extract better contextual input frames. Note that, different from the previous DNN/DMDN architectures, in the proposed RNN-based spectral conversion models, all of the components, except for the normalization and denormalization layers (which are kept fixed to make the network work with the normalized values of the features), are optimized during training.

\section{1) LSTM-BASED SPECTRAL CONVERSION MODEL}

Given an input spectral feature vector $\boldsymbol{x}_{t}$ at frame $t$, the estimated target spectral feature vector $\hat{\boldsymbol{y}}_{t}$ is estimated as

$$
\hat{\boldsymbol{y}}_{t}=\boldsymbol{W}_{h y} \boldsymbol{h}_{t}+\boldsymbol{b}_{y}
$$

where $\boldsymbol{W}_{h y}$ and $\boldsymbol{b}_{y}$, respectively, denote the weights and biases of the output layer in Fig 2. The hidden state $\boldsymbol{h}_{t}$ is produced by the LSTM units [36] as follows:

$$
\begin{aligned}
\boldsymbol{i}_{t} & =\sigma\left(\boldsymbol{W}_{x i}\left[\boldsymbol{x}_{t}^{\top}, \hat{\boldsymbol{y}}_{t-1}^{\top}\right]^{\top}+\boldsymbol{W}_{h i} \boldsymbol{h}_{t-1}+\boldsymbol{b}_{i}\right) \\
\boldsymbol{f}_{t} & =\sigma\left(\boldsymbol{W}_{x f}\left[\boldsymbol{x}_{t}^{\top}, \hat{\boldsymbol{y}}_{t-1}^{\top}\right]^{\top}+\boldsymbol{W}_{h f} \boldsymbol{h}_{t-1}+\boldsymbol{b}_{f}\right) \\
\boldsymbol{g}_{t} & =\tanh \left(\boldsymbol{W}_{x g}\left[\boldsymbol{x}_{t}^{\top}, \hat{\boldsymbol{y}}_{t-1}^{\top}\right]^{\top}+\boldsymbol{W}_{h g} \boldsymbol{h}_{t-1}+\boldsymbol{b}_{g}\right) \\
\boldsymbol{o}_{t} & =\sigma\left(\boldsymbol{W}_{x o}\left[\boldsymbol{x}_{t}^{\top}, \hat{\boldsymbol{y}}_{t-1}^{\top}\right]^{\top}+\boldsymbol{W}_{h o} \boldsymbol{h}_{t-1}+\boldsymbol{b}_{o}\right) \\
\boldsymbol{c}_{t} & =\boldsymbol{f}_{t} \odot \boldsymbol{c}_{t-1}+\boldsymbol{i}_{t} \odot \boldsymbol{g}_{t} \\
\boldsymbol{h}_{t} & =\boldsymbol{o}_{t} \odot \tanh \left(\boldsymbol{c}_{t}\right)
\end{aligned}
$$

where $\hat{\boldsymbol{y}}_{\mathrm{o}}=\mathbf{o}$. The cell state is denoted as $\boldsymbol{c}_{t}$. The input, forget, cell, and output gates are denoted as $\boldsymbol{i}_{t}, \boldsymbol{f}_{t}, \boldsymbol{g}_{t}$, and $\boldsymbol{o}_{t}$, respectively. The trainable weights and biases are denoted by the corresponding $\boldsymbol{W}$ and $\boldsymbol{b}$, respectively. $\sigma$ denotes a sigmoid function and $\odot$ denotes an element-wise product.
The network model parameters $\hat{\lambda}_{\mathrm{R}}$ are optimized through backpropagation for the loss function, computed by considering the distortions in the mel-cepstrum domain [54], as follows:

$$
\hat{\lambda}_{\mathrm{R}}=\arg \min _{\lambda_{\mathrm{R}}} \frac{1}{T} \sum_{t=1}^{T} \frac{10 \sqrt{2}}{\ln 10} \sum_{d=1}^{D}\left|\hat{y}_{t}(d)-y_{t}(d)\right|,
$$

where $|\cdot|$ denotes the absolute function. The $d$ th dimension estimated spectral feature at time $t$ is denoted as $\hat{y}_{t}(d)$, where the estimated spectral feature vector $\hat{\boldsymbol{y}}_{t}$ can be written in terms of the feedforward function in an RNN-based spectral mapping model as $f_{\lambda_{R}}\left(\boldsymbol{x}_{t}\right)=$ $\left[\hat{y}_{t}(1), \hat{y}_{t}(2), \ldots, \hat{y}_{t}(d), \ldots, \hat{y}_{t}(D)\right]^{\top}$. Note that the input source spectra $\boldsymbol{x}_{t}$ is firstly fed into the input convolutional layers before being fed into the RNN units as shown in Fig. 2.

\section{2) GRU-BASED SPECTRAL CONVERSION MODEL}

In addition to modeling the long-term context dependencies, it is also worthwhile considering a more compact model architecture, such as the GRU [37] units, which will be more suitable for our purpose in using a small amount of training data. A more compact network will also be more suitable for real-time applications, which require lowlatency computation.

In the proposed GRU-based spectral conversion model, given an input spectral feature vector $\boldsymbol{x}_{t}$ at frame $t$, the following functions are computed by the GRU units:

$$
\begin{aligned}
& \boldsymbol{r}_{t}=\sigma\left(\boldsymbol{W}_{x r}\left[\boldsymbol{x}_{t}, \hat{\boldsymbol{y}}_{t-1}^{\top}\right]^{\top}+\boldsymbol{W}_{h r} \boldsymbol{h}_{t-1}+\boldsymbol{b}_{r}\right) \\
& \boldsymbol{z}_{t}=\sigma\left(\boldsymbol{W}_{x z}\left[\boldsymbol{x}_{t}, \hat{\boldsymbol{y}}_{t-1}^{\top}\right]^{\top}+\boldsymbol{W}_{h z} \boldsymbol{h}_{t-1}+\boldsymbol{b}_{z}\right) \\
& \boldsymbol{n}_{t}=\tanh \left(\boldsymbol{W}_{x n}\left[\boldsymbol{x}_{t}, \hat{\boldsymbol{y}}_{t-1}^{\top}\right]^{\top}+\boldsymbol{r}_{t} \odot\left(\boldsymbol{W}_{h n} \boldsymbol{h}_{t-1}\right)+\boldsymbol{b}_{n}\right) \\
& \boldsymbol{h}_{t}=\left(1-\boldsymbol{z}_{t}\right) \odot \boldsymbol{n}_{t}+\boldsymbol{z}_{t} \odot \boldsymbol{h}_{t-1},
\end{aligned}
$$

where $\hat{\boldsymbol{y}}_{t}$ is given by (8) and, similarly, $\hat{\boldsymbol{y}}_{\mathrm{o}}=\mathbf{o}$. The hidden state of the GRU is denoted as $\boldsymbol{h}_{t}$. The reset, update, and new gates are denoted as $\boldsymbol{r}_{t}, \boldsymbol{z}_{t}$, and $\boldsymbol{n}_{t}$, respectively. The corresponding weights and biases are, respectively, denoted by $W$ and $\boldsymbol{b}$. The sigmoid function is denoted by $\sigma$ and $\odot$ denotes an element-wise product. As in the LSTM-based model, for multiple layers of GRU units, the hidden state of the current layer is used as the input for the succeeding layer. As 


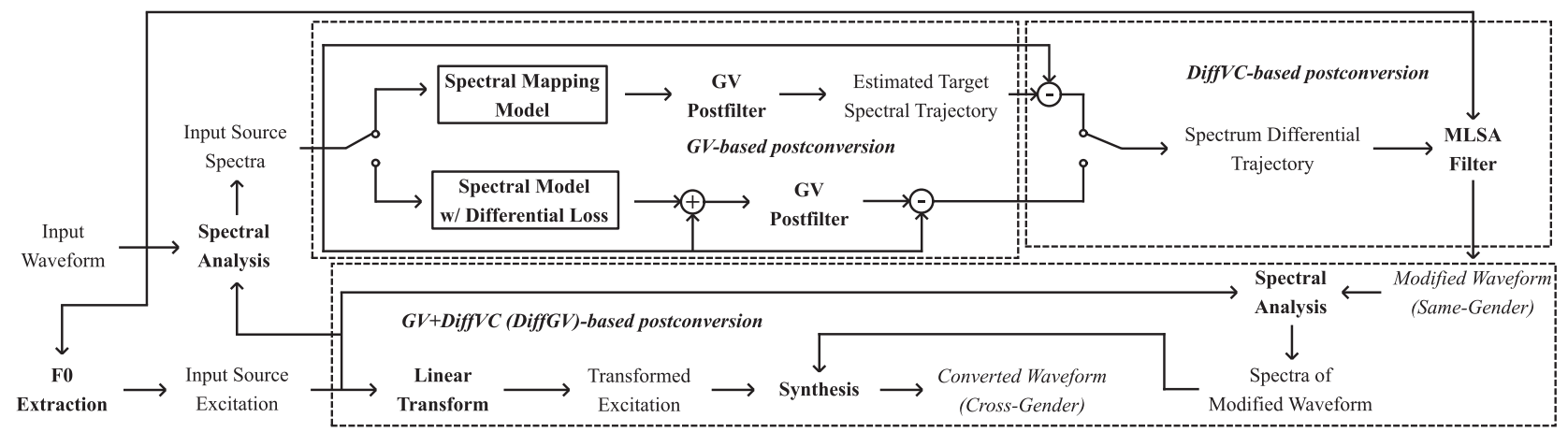

Fig. 3. Flow of direct waveform modification with spectrum differential (DiffVC) [5] using MLSA synthesis filter [55] and GV postfilter [22].

can be observed, the modeling of long-term context dependencies in GRU is achieved through fewer computation steps, and model parameters, compared to the LSTM, which might be better for limited data situations and real-time computation.

\section{WAVEFORM GENERATION MODELS WITH WN VOCODER}

In this paper, in addition to the spectral conversion models, we also employ an NN-based architecture for the waveform generator (neural vocoder), i.e. the $\mathrm{WN}[23]$ model. As a first step towards the generalization of the use of other neural vocoders in $\mathrm{VC}$, we describe the use of $\mathrm{WN}$ vocoder [44-46], where the WN model is conditioned using both speech waveform samples and extracted speech parameters. We address the quality degradation problem faced by the $\mathrm{WN}$ vocoder in $\mathrm{VC}$ when it utilizes estimated speech parameters [47] from a spectral conversion model, through a post-conversion processing [24]. Finally, we propose a modified loss function in the development of the spectral conversion model to preserve the consistency with the waveform generation procedure.

\section{A) $\mathrm{WN}$ vocoder}

$\mathrm{WN}$ [23] is a deep AR-CNN that is capable of modeling speech waveform samples for their previous samples. To efficiently increase the number of receptive fields of waveform samples, WN uses a stack of dilated CNNs using residual blocks, making it possible to produce human-like speech sounds. Moreover, when it is conditioned with naturally extracted speech features, such as spectral and excitation parameters, a WN vocoder [44-46] is capable of producing meaningful speech waveforms with the natural quality compared with the conventional vocoder framework.

Given a sequence of auxiliary feature vectors $\boldsymbol{h}=\left[\boldsymbol{h}_{1}^{\top}, \boldsymbol{h}_{2}^{\top}, \ldots, \boldsymbol{h}_{t}^{\top}, \ldots, \boldsymbol{h}_{T}^{\top}\right]^{\top}$, the likelihood function of a sequence of the corresponding waveform samples $\boldsymbol{s}=\left[s_{1}, s_{2}, \ldots, s_{t}, \ldots, s_{T}\right]^{\top}$ is given by

$$
P(\boldsymbol{s} \mid \boldsymbol{h}, \boldsymbol{\lambda})=\prod_{t=1}^{T} P\left(s_{t} \mid \boldsymbol{h}_{t}, \boldsymbol{s}_{t-p}\right),
$$

where the conditional PDF of a waveform sample $P\left(s_{t} \mid \boldsymbol{h}_{t}, \boldsymbol{s}_{t-p}\right)$ is modeled by the WN vocoder, and $\boldsymbol{s}_{t-p}$ denotes the previous samples with $p$ receptive fields. In the training, the ground-truth previous samples are given, i.e. teacher-forcing mode, whereas, in the synthesis phase, the waveform is generated sample-by-sample. Note that the modeling of the waveform samples in a $\mathrm{WN}$ vocoder can be performed as a classification problem, where the floating values of the 16-bit waveform will be discretized into 256 categories using the $\mu$-law algorithm. The inverse $\mu$-law algorithm is used in synthesis after sampling from the output distribution.

\section{B) Postconversion processing in $\mathrm{VC}$}

In $\mathrm{VC}$, to use the $\mathrm{WN}$ vocoder, estimated speech features, generated from a statistical mapping model, such as the ones described in Section III, are fed as auxiliary conditioning features in equation (20) to generate the converted speech waveform. However, in this case, the $\mathrm{WN}$ vocoder will face a significant degradation [47], because of the mismatches of speech features, such as the oversmoothed characteristics of the estimated spectral features due to the mean-based optimization of a spectral conversion model. To improve the quality of the converted speech waveform, these mismatches between the naturally extracted speech parameters and the oversmoothed estimated speech parameters have to be alleviated, e.g. with a post-conversion processing method.

The ideal way to address the degradation problem of WN vocoder in VC is by directly addressing the mismatches of speech features in the development of a $\mathrm{WN}$ vocoder. In this work, as a first step toward improving the use of the neural vocoder in VC, we propose to overcome the quality degradation by the use of post-conversion processing to alleviate the feature mismatches, such as oversmoothing problem. The proposed post-conversion processing is based on GV [22] postfilter and DiffVC [5], which is illustrated in Fig. 3. The GV postfilter is used to recover the variances of the oversmoothed spectral features to make them closer to the natural ones. The DiffVC method is used to further reduce the mismatches between the spectral and excitation features by directly modifying the input speech waveform according to the differences between the estimated target spectra 


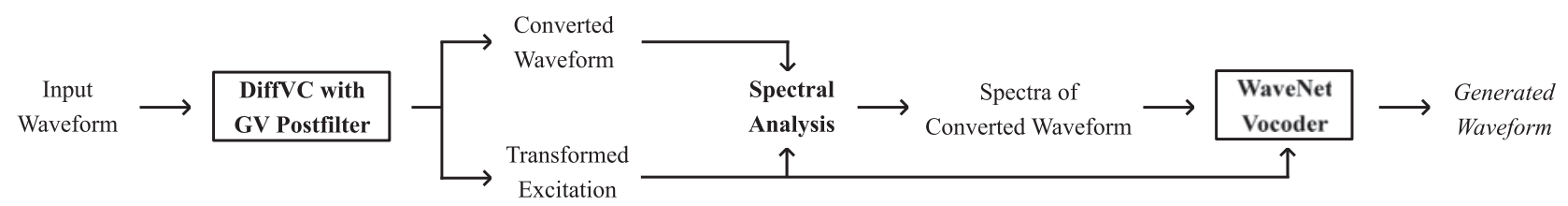

Fig. 4. Waveform generation procedure for converted speech using WN vocoder through the use of post-conversion processed auxiliary speech features based on the DiffVC method and the GV postfilter (DiffGV).

and the input spectra. Then, through the combination of GV and DiffVC post-conversion processing (DiffGV), the modified set of speech parameters [24] can be obtained for use in the WN vocoder as shown in Fig. 4. Note that, as shown in Fig. 3, for cross-gender conversions, e.g. maleto-female speakers, an additional step of analysis-synthesis is performed with also the transformed excitation features because the DiffVC [5] method does not modify the original excitation when filtering to generate the modified waveform using the mel-log spectrum approximation (MLSA) filter [55].

Finally, to preserve the consistency between the spectral conversion model and the waveform generation procedure, due to the use of spectrum differential method (DiffVC), we propose the use of an alternative loss function for the RNNbased architectures that considers the spectrum differences. Given the estimated feature vector $\hat{\boldsymbol{y}}_{t}=f_{\lambda_{\mathrm{R}}}\left(\boldsymbol{x}_{t}\right)$ from (8), instead of optimizing the parameters with respect to the ground truth $\boldsymbol{y}_{t}$, the optimization is performed as follows:

$$
\hat{\lambda}_{\mathrm{R}}=\arg \min _{\lambda_{\mathrm{R}}} \frac{1}{T} \sum_{t=1}^{T} \frac{10 \sqrt{2}}{\ln 10} \sum_{d=1}^{D}\left|\hat{y}_{t}(d)-\left(y_{t}(d)-x_{t}(d)\right)\right|,
$$

where $f_{\lambda_{R}}(\cdot)$ is the feedforward function using the RNNbased spectral conversion model, as described in Section 3.3, and $|\cdot|$ denotes the absolute function. Therefore, the conversion model is optimized to directly generate the spectrum differential trajectory, i.e. the trajectory difference between the target $y$ and the source spectra $x$, which is used to generate the set of post-conversion processed speech features for generating the converted speech waveform with the $\mathrm{WN}$ vocoder. As a further note, the constant coefficient in (15) and (21) comes from the MCD formulation [54], which is quite effective in our experimental conditions on the RNN-based spectral modeling.

\section{EXPERIMENTAL EVALUATION}

\section{A) Experimental conditions}

In the experiments, we used the VCC 2018 dataset [51], consisting of six female and six male speakers, as well as additional data from the CMU Arctic dataset [56], where we used the data of "bdl" (male) and "slt" (female) speakers to develop a multispeaker WN vocoder [45]. On the other hand, to develop the spectral conversion models, we used a subset of the VCC 2018 data, comprising "SF1" and "SM1" data as the source speakers, as well as "TF1" and "TM1" data as the target speakers, where " $F$ " means female and " $M$ " means male, giving a total of four conversion models for each of the network architectures described in Section III. The total numbers of utterances in the CMU Arctic dataset and VCC 2018 dataset are 1132 and 81, respectively. The training set from the CMU Arctic utterances consisted of the first 992 utterances, whereas that from the VCC 2018 dataset consisted of the final 71 utterances. The remaining sentences in both datasets were used as testing data. The number of evaluation utterances provided in the VCC 2018 dataset is 35 , which were used in the subjective evaluation. The length of each audio sample in the training data was roughly $3.5 \mathrm{~s}$ on average.

As the spectral features, we used the $34 \mathrm{D}$ mel-cepstrum parameters, including the oth power coefficient, extracted from the spectral envelope of the WORLD spectrum $[38,57]$. The frequency warping parameter was set to 0.455 [58]. The spectral envelope of the speech spectrum was computed frame by frame using CheapTrick $[59,60]$ and then parameterized into the mel-cepstrum coefficients [58]. We used framewise $F_{0}$ values as the excitation features as well as the two-band aperiodicity features, which were, respectively, extracted using Harvest [61] and $\mathrm{D}_{4} \mathrm{C}[62]$ in the WORLD package. To perform the prosody conversion, we carried out a linear transformation of the $F_{\mathrm{o}}$ values using the statistics of the source and target speakers. For the set of auxiliary speech parameters used in the $\mathrm{WN}$ vocoder, we utilized continuous interpolated $F_{\mathrm{o}}$ values and binary unvoiced/voiced (U/V) decisions, giving $39 \mathrm{D}$ auxiliary speech parameters, i.e. $1 \mathrm{D} U / \mathrm{V}, 1 \mathrm{D}$ continuous Fo, $2 \mathrm{D}$ code-aperiodicity, oth power, and $34 \mathrm{D}$ mel-cepstrum. The speech signal sampling rate was $22,050 \mathrm{kHz}$ and the frameshift was set to $5 \mathrm{~ms}$.

The WN architecture comprised a 1,2,4, ., 1024 dilation sequence with four repetitions. The number of residual channels was 128 and the number of skip channels was 256. Two convolution layers with ReLU activation were used after the skip connections before the softmax output layer. The trained multispeaker $\mathrm{WN}$ model was finetuned for each target speaker, i.e. TF1 or TM1, using their extracted speech parameters. The implementation of $\mathrm{WN}$ was based on [63], where the noise-shaping method [64] was also used. To train the WN model parameters, the Adam algorithm [65] was employed. The weights of model parameters were initialized using Xavier [66], while the biases were zero-initialized. The learning rate was set to o.ooo1. The hyperparameters for $\mathrm{WN}$ training, i.e. the optimization algorithm, the initialization method, and the learning rate, were set to the same as those for the training of spectral conversion models. 
In the development of the spectral conversion models, we used five hidden layers for the DNN model and four hidden layers for the DMDN model with ReLU activation functions. The number of mixture components for the DMDN was set to 16. For the RNN-based models, we used one layer for both hidden LSTM/GRU units. Convolutional input layers were used for all DNN-/DMDN-/RNN-based spectral models, where they were designed to capture four preceding and four succeeding frame input contexts with dynamic dimensions. Specifically, two convolutional input layers were used with a kernel size of 3 and a dilation size of 1 and 3, respectively, for each layer. The number of output dimensions for each layer was its kernel size multiplied by the number of input dimensions, e.g. with $50 \mathrm{D}$ features, the first input layer will have $150 \mathrm{OD}$ output and the second layer will have $450 \mathrm{D}$ output. Dropout [67] layers with 0.5 probability were used in the training of the RNN-based models after the convolutional input layers and after the hidden LSTM/GRU units. For the RNN-based methods, we also trained additional models using the proposed loss for the spectrum differential in (21). An additional normalization layer before the input convolutional layers and a de-normalization layer after the final output layer were used and fixed according to the statistics obtained from the training data. The performance of all conversion models was compared in the objective evaluation. In the subjective evaluation, we used the GRU-based conversion model with both the conventional loss in (15) and the proposed spectrum differential loss in (21). The dynamic-time-warping procedure was used to create time-warping functions for computing the loss between the estimated target spectra and the ground truth by only using the speech frames (non-silent frames).

\section{B) Objective evaluation}

To objectively evaluate the NN-based spectral mapping models described in Section III, we computed the metrics of MCD [22, 54] and LGD. These metrics were computed between the converted mel-cepstrum parameters of the source speaker and the extracted (natural) mel-cepstrum parameters of the target speaker for all speaker pairs, i.e. $\mathrm{SF}_{1}-\mathrm{TF} 1, \mathrm{SF}_{1}-\mathrm{TM} 1, \mathrm{SM}_{1}-\mathrm{TF}$, and $\mathrm{SM}_{1}-\mathrm{TM} 1$.

The MCD was computed using

$$
\mathrm{MCD}[\mathrm{dB}]=\frac{1}{T} \sum_{t=1}^{T} \frac{10}{\ln 10} \sqrt{2 \sum_{d=2}^{D}\left(\hat{y}_{t}(d)-y_{t}(d)\right)^{2}},
$$

where $\hat{y}_{t}(d)$ is the $d$ th dimension of the converted melcepstrum and $y_{t}(d)$ is that of the target mel-cepstrum at frame $t$. The starting dimension $d$ is set to 2 for only measuring the mel-cepstrum parameters without the oth power. On the other hand, to compute the LGD, the following function was used:

$$
\mathrm{LGD}=\frac{1}{D} \sum_{d=2}^{D} \sqrt{\left(\log \sigma_{\text {global }}(\hat{y}(d))-\log \sigma_{\text {global }}(y(d))\right)^{2}},
$$

where the $\mathrm{GV}[22] \sigma_{\text {global }}(\cdot)$ is computed as

$$
\sigma_{\text {global }}(y(d))=\frac{1}{N} \sum_{n=1}^{N} \frac{1}{T_{n}} \sum_{t=1}^{T_{n}}\left(y_{t}(d)-\bar{y}_{n}(d)\right)^{2} .
$$

The index of the $n$th training data is denoted by $n$, the total number of training sentences is $N$, and the mean value of the $d$ th dimension target spectra of the $n$th utterance is denoted as $\bar{y}_{n}(d)$. The objective measurements of all the spectral conversion models were computed. These models consisted of the DNN models, the DMDN models, the LSTM-based models, the GRU-based models with conventional loss, and the GRU-based models with the proposed spectrum differential loss in (21) (GRUDiff).

The results for MCD measurements of the training and testing data, averaged over all speaker-pair conversions, are shown in Figs 5 and 6, respectively, during 500 training epochs for DNN/DMDN models, and during 325 epochs for LSTM/GRU/GRUDiff models. The lower number of epochs for RNN-based models is due to their faster convergence rate compared to the conventional DNN-/DMDN-based models. It can be observed that the GRU-based spectral models with conventional loss give better accuracy and generalization for unseen data, where it yields higher accuracy than the other models within only 50-80 epochs. Similar tendencies are also observed for the same- and cross-gender conversions, as, respectively, shown in Figs 7 and 8. Note that the lower accuracy for the GRUDiff models is due to the optimization for generating the spectrum differential rather than the target spectra. Although the MCD measurement does not always correlate with perceptual output, they can still be used as a basic metric for monitoring the convergence and the basic performance of spectral mapping models. As a further side note, for the training time, one epoch of the DNN/DMDN-based network takes about two times faster than the LSTM-/GRU-based network. However, the convergence of the LSTM-/GRU-based network takes only about 50-80 epochs, whereas that of the DNN/DMDN takes nearly 500 epochs.

Hence, to accompany the pure accuracy results of MCD, the LGD measurements were performed to evaluate the oversmoothness (variance reduction) of spectral trajectory due to the mean-based optimization in spectral modeling. The results of the LGD measurements of the testing data for same- and cross-gender conversions are given in Figs 9 and 10, respectively. These results demonstrate that the proposed spectrum differential loss used for the GRUDiff models makes it possible for the resulting converted trajectory to be less oversmoothed. Furthermore, all of the RNN-based spectral models show a clear tendency of being capable of producing better trajectory variance compared to the $\mathrm{DNN}$ /DMDN-based models, such as the one used in our previous work [24], i.e. DMDN-based model. Considering the capability of GRU and GRUDiff to produce superior objective results, we used them both to generate converted spectra in the subjective evaluation. As to the latter point, in this work, we indeed focus on providing empirical evidence of the performance of RNN-based architectures for the VC 


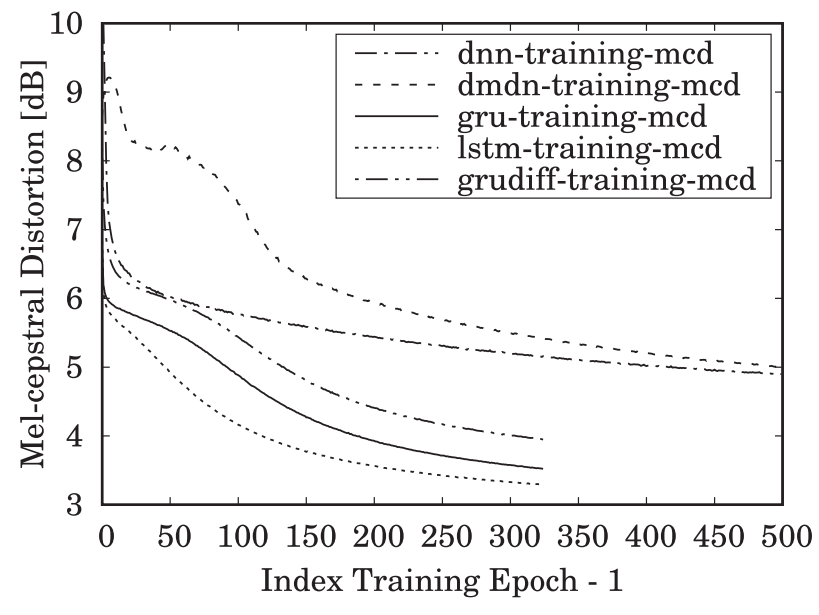

Fig. 5. Trend of mel-cepstral distortion (MCD) for the training set using the DNN-, DMDN-, LSTM-, GRU-, and GRUDiff-based spectral conversion models during 500 training epochs for the DNN/DMDN models and 325 training epochs for the LSTM/GRU/GRUDiff models.

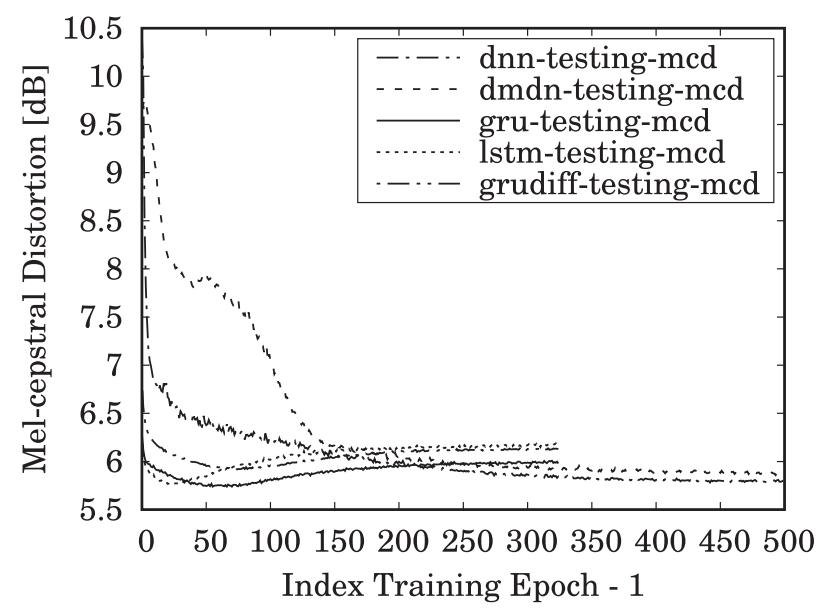

Fig. 6. Trend of MCD for the testing set using the DNN-, DMDN-, LSTM-, GRU-, and GRUDiff-based spectral conversion models during 500 training epochs for the DNN/DMDN models and 325 training epochs for the LSTM/GRU/GRUDiff models.

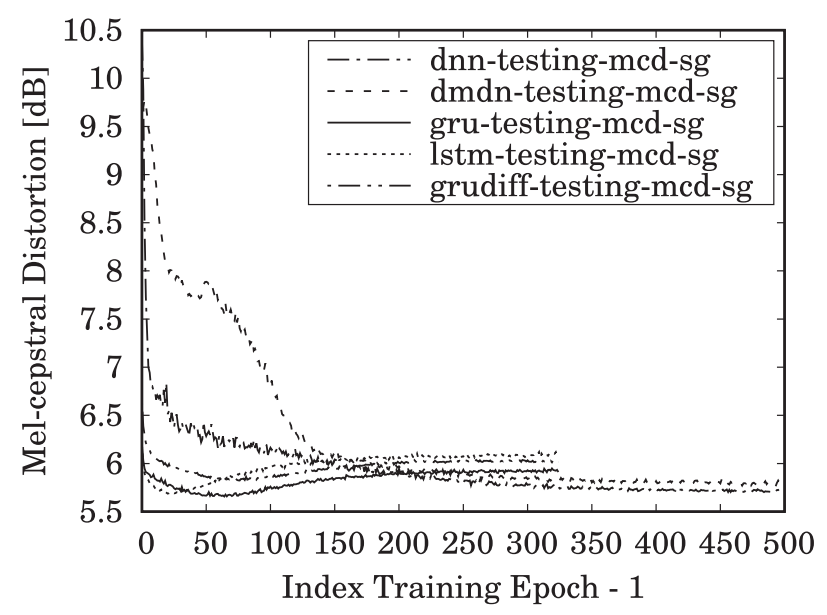

Fig. 7. Trend of MCD for same-gender (SG) conversions on the testing set using the DNN-, DMDN-, LSTM-, GRU-, and GRUDiff-based spectral conversion models during 500 training epochs for the DNN/DMDN models and 325 training epochs for the LSTM/GRU/GRUDiff models.

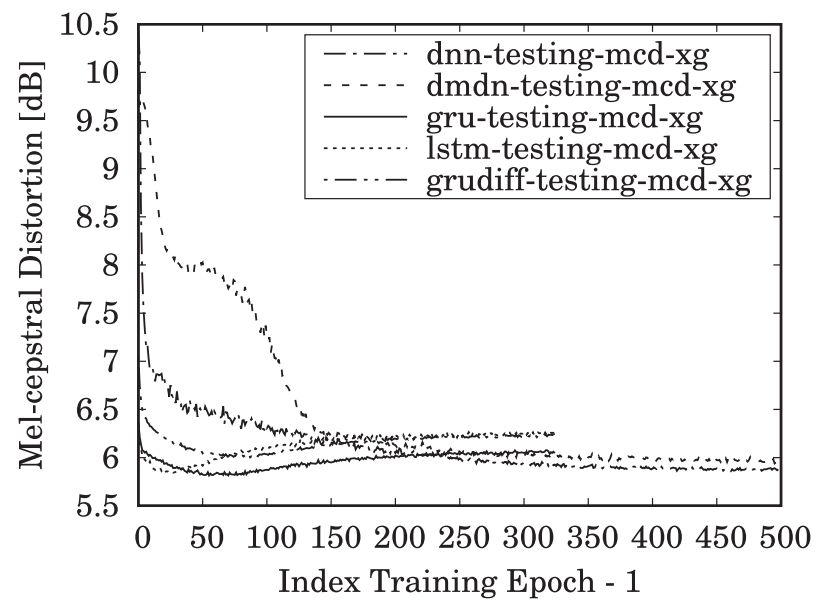

Fig. 8. Trend of MCD for cross-gender (XG) conversions on the testing set using the DNN-, DMDN-, LSTM-, GRU-, and GRUDiff-based spectral conversion models during 500 training epochs for the DNN/DMDN models and 325 training epochs for the LSTM/GRU/GRUDiff models.

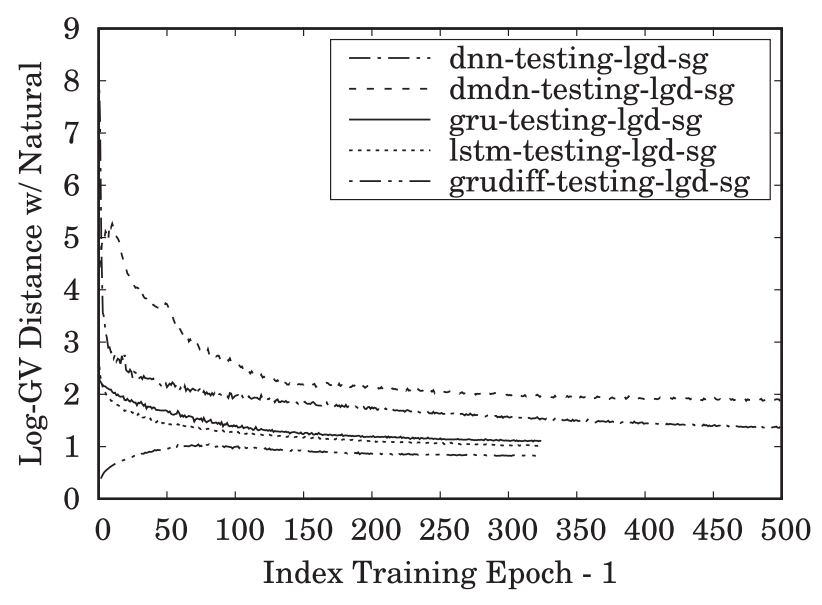

Fig. 9. Trend of log-GV distance (LGD) for SG conversions on the testing set using the DNN-, DMDN-, LSTM-, GRU-, and GRUDiff-based spectral conversion models during 500 training epochs for the DNN/DMDN models and 325 training epochs for the LSTM/GRU/GRUDiff models.

framework, which would be beneficial for the continuous future development of real-world applications and integration of training development with other frameworks, such as neural vocoder.

\section{C) Subjective evaluation}

In the subjective evaluation, we conducted MOS tests to evaluate the naturalness of the converted speech waveforms and speaker similarity tests to evaluate the accuracy of the converted speech waveforms. In the MOS tests, a five-scale score was used to assess the naturalness of speech utterances, i.e. 1: completely unnatural, 2: mostly unnatural, 3: equally natural and unnatural, 4: mostly natural, 5: completely natural. On the other hand, for the speaker similarity tests, each listener was given a pair of stimuli, i.e. a natural speech of the target speaker and a converted speech of the source speaker, and asked to judge whether or not the two speech utterances were produced by the same speaker. To 


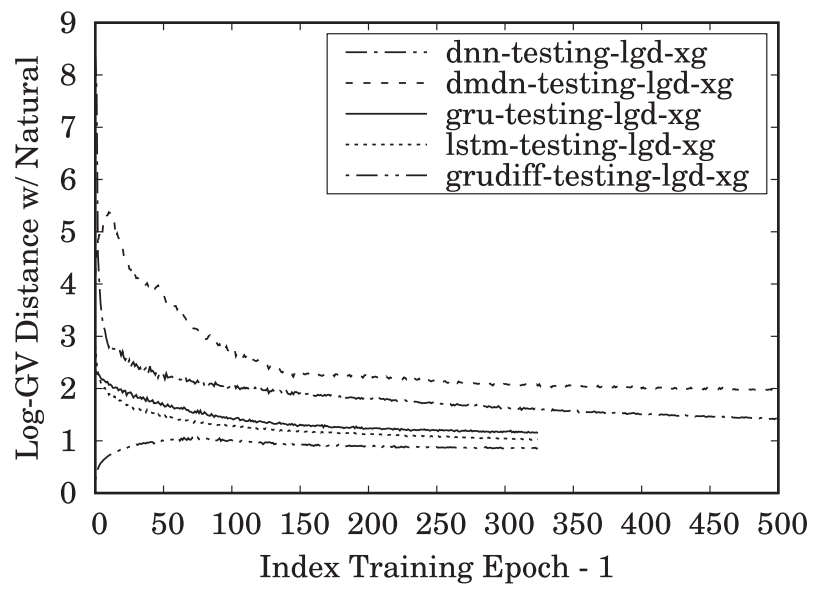

Fig. 10. Trend of LGD for XG conversions on the testing set using the DNN-, DMDN-, LSTM-, GRU-, and GRUDiff-based spectral conversion models during 500 training epochs for the DNN/DMDN models and 325 training epochs for the LSTM/GRU/GRUDiff models.

judge the similarity, each listener chose between two main responses, i.e. "same" or "different", where each had a confidence measurement, i.e. "sure" or "not sure", giving a total of four options when judging the similarity. The number of listeners was 10.

We compared the use of direct waveform modification with the spectrum differential, i.e. DiffVC [5], using the GV [22] postfilter (dG), which was made to nearly matching the baseline system of VCC 2018 [68], and the use of the $\mathrm{WN}$ vocoder to generate the converted speech waveforms. In the case of the WN-based waveform generation, we also compared the use of several types of speech auxiliary features, namely, converted spectral features (WNc), converted features with the GV postfilter (WNcG), postconversion processing based on DiffVC (WNd), and DiffVC post-conversion processing with the GV postfilter (WNdG), which was used for our VC system in VCC 2018 [24]. Furthermore, we also utilized two different spectral mapping models, i.e. the GRU-based models with conventional loss (GRU) and spectrum differential loss (GRUDiff). The total combinations of speaker pairs and spectral mapping models/waveform generation methods were 40, i.e. four speaker pairs and ten different models/methods. In the naturalness evaluation, the number of distinct utterances was three, whereas, in the speaker similarity evaluation, it was two. In both evaluations, we also included the original speech waveform of the four speakers, i.e. either as a single stimulus of the original waveform in naturalness test or a pair of stimuli of original waveforms (from either source-target/target-target) in similarity test. Therefore, each listener had to evaluate 132 audio samples in the naturalness test and 92 audio samples in the similarity test.

The results of the MOS tests are shown in Tables 1 and 2. It can be observed the DiffVC with the GV method yields relatively high naturalness scores for same-gender conversions but not for cross-gender conversions owing to the use of the conventional vocoder in the cross-gender conversions. On the other hand, for the $\mathrm{WN}$-based waveform generation, the use of post-conversion processing based on DiffVC and the GV, i.e. WNdG, clearly enhances the naturalness of the converted speech waveforms compared with the other sets of auxiliary speech features. Furthermore, the proposed spectrum differential loss improves the performance for same-gender conversions, i.e. either F-to-F or M-to-M.

Results for the speaker similarity tests are given in Table 3. The results show that the use of the WN vocoder with the DiffGV-based post-conversion processed auxiliary features (WNdG) yields superior accuracy to the other methods, including the conventional DiffGV without the $\mathrm{WN}$ vocoder $(\mathrm{dG})$. The results for naturalness and accuracy in this paper exhibit a similar tendency to the results of VCC 2018 [51], where the baseline method [68] yields better naturalness, especially for same-gender conversions, due to the avoidance of the conventional vocoder, and our $\mathrm{VC}$ system with post-conversion processing for the $\mathrm{WN}$ vocoder [24] gives better accuracy. Based on these results, for the use of a WN vocoder in VC, it has been shown that an additional processing procedure adjusted to reduce the mismatches between estimated and natural speech features is needed to improve the converted speech waveform. For future work, it is worthwhile to directly address this problem in the development of a neural vocoder, such as $\mathrm{WN}$, for $\mathrm{VC}$, i.e. in a data-driven manner. All audio samples used in the subjective evaluation are available at http://bit.ly/2WjuJd1.

Table 1. Results of mean opinion score (MOS) test of DiffVC with the GV postfilter (dG) waveform generation method using either GRU or GRUDiff spectral mapping models and of the original speech signals.

\begin{tabular}{|c|c|c|c|c|c|c|}
\hline \multirow[b]{2}{*}{ MOS } & \multirow{2}{*}{$\begin{array}{l}\text { GRU } \\
\text { dG }\end{array}$} & \multirow{2}{*}{$\begin{array}{l}\text { GRUDiff } \\
\text { dG }\end{array}$} & \multicolumn{4}{|c|}{ Original } \\
\hline & & & $\mathrm{SF}_{1}$ & SM1 & TF1 & TM1 \\
\hline All pairs & $3.27 \pm 0.22$ & $3.28 \pm 0.23$ & $4.93 \pm 0.09$ & $5.00 \pm 0.00$ & $4.93 \pm 0.07$ & $4.87 \pm 0.09$ \\
\hline S-Gender & $4.08 \pm 0.24$ & $4.33 \pm 0.18$ & - & - & - & - \\
\hline $\mathrm{SF}_{1}-\mathrm{TF}_{1}$ & $4.10 \pm 0.37$ & $4.20 \pm 0.25$ & - & - & - & - \\
\hline SM1-TM1 & $4.07 \pm 0.31$ & $4.47 \pm 0.25$ & - & - & - & - \\
\hline X-Gender & $2.45 \pm 0.24$ & $2.23 \pm 0.21$ & - & - & - & - \\
\hline SF1-TM1 & $2.00 \pm 0.33$ & $1.80 \pm 0.25$ & - & - & - & - \\
\hline $\mathrm{SM}_{1}-\mathrm{TF}_{1}$ & $2.90 \pm 0.28$ & $2.67 \pm 0.27$ & - & - & - & - \\
\hline
\end{tabular}

\pm denotes the $95 \%$ confidence interval. S-Gender and X-Gender denote same-gender and cross-gender conversions, respectively. 
Table 2. Results of MOS test of the WN-based generation methods using plain converted mel-cepstrum (c), using c with GV postfilter (cG), using post-conversion based on DiffVC (d), and using d with GV postfilter (dG) from either GRU or GRUDiff spectral mappings.

\begin{tabular}{|c|c|c|c|c|c|c|c|c|}
\hline \multirow[b]{2}{*}{ MOS } & \multicolumn{4}{|c|}{ GRU } & \multicolumn{4}{|c|}{ GRUDiff } \\
\hline & $\mathrm{WNc}$ & $\mathrm{WNcG}$ & WNd & WNdG & WNc & WNcG & WNd & WNdG \\
\hline All pairs & {$[2.35 \pm 0.16]$} & {$[2.56 \pm 0.17]$} & {$[2.53 \pm 0.18]$} & $2.99 \pm 0.18$ & {$[2.62 \pm 0.19]$} & {$[2.71 \pm 0.19]$} & {$[2.72 \pm 0.19]$} & $2.98 \pm 0.18$ \\
\hline S-Gender & {$[2.47 \pm 0.23]$} & {$[2.70 \pm 0.26]$} & {$[2.77 \pm 0.25]$} & $3.25 \pm 0.25$ & $3.05 \pm 0.26$ & $3.18 \pm 0.26$ & $2.92 \pm 0.29$ & $3.30 \pm 0.25$ \\
\hline $\mathrm{SF}_{1}-\mathrm{TF}_{1}$ & {$[2.43 \pm 0.34]$} & {$[2.70 \pm 0.36]$} & $2.93 \pm 0.35$ & $3.33 \pm 0.37$ & {$[2.70 \pm 0.26]$} & {$[2.83 \pm 0.28]$} & $2.97 \pm 0.40$ & $3.37 \pm 0.36$ \\
\hline $\mathrm{SM}_{1}-\mathrm{TM} 1$ & {$[2.50 \pm 0.34]$} & {$[2.70 \pm 0.39]$} & {$[2.60 \pm 0.38]$} & $3.17 \pm 0.36$ & $3.40 \pm 0.42$ & $3.53 \pm 0.40$ & {$[2.87 \pm 0.44]$} & $3.23 \pm 0.28$ \\
\hline X-Gender & {$[2.23 \pm 0.23]$} & $2.42 \pm 0.23$ & {$[2.28 \pm 0.24]$} & $2.73 \pm 0.25$ & {$[2.18 \pm 0.22]$} & {$[2.23 \pm 0.23]$} & $2.52 \pm 0.23$ & $2.65 \pm 0.24$ \\
\hline $\mathrm{SF}_{1}-\mathrm{TM} 1$ & {$[2.20 \pm 0.39]$} & $2.73 \pm 0.35$ & $2.47 \pm 0.35$ & $2.60 \pm 0.35$ & {$[2.20 \pm 0.35]$} & $2.30 \pm 0.39$ & {$[2.23 \pm 0.32]$} & $2.43 \pm 0.39$ \\
\hline $\mathrm{SM}_{1}-\mathrm{TF}_{1}$ & {$[2.27 \pm 0.26]$} & {$[2.10 \pm 0.27]$} & {$[2.10 \pm 0.33]$} & $2.87 \pm 0.38$ & {$[2.17 \pm 0.30]$} & {$[2.17 \pm 0.26]$} & $2.80 \pm 0.32$ & $2.87 \pm 0.29$ \\
\hline
\end{tabular}

S-Gender and X-Gender, respectively, denote same-gender and cross-gender conversions. \pm denotes the $95 \%$ confidence interval of the sample mean. Bold indicates the system(s) with the highest mean score in each conversion category. [.] Denotes a system with a statistically significant lower score than the highest score in each conversion category. Statistical inferences were performed using the two-tailed Mann-Whitney test with $\alpha<0.05$.

Table 3. Results of speaker similarity test (scores were aggregations of "same - sure" and "same - not sure" decisions) of the converted speech waveform using all waveform generation methods ( $\mathrm{dG}, \mathrm{WNc}, \mathrm{WNcG}, \mathrm{WNd}$, and $\mathrm{WNdG}$ ) with either GRU or GRUDiff spectral mappings.

\begin{tabular}{|c|c|c|c|c|c|c|c|c|c|c|}
\hline \multirow[b]{2}{*}{ Speaker similarity scores (\%) } & \multicolumn{5}{|c|}{ GRU } & \multicolumn{5}{|c|}{ GRUDiff } \\
\hline & dG & WNc & WNcG & WNd & WNdG & dG & WNc & WNcG & WNd & WNdG \\
\hline All pairs & 66.25 & 57.50 & [58.75] & 65.00 & 71.25 & {$[46.25]$} & [57.50] & [52.50] & 61.25 & 63.75 \\
\hline S-Gender & 82.50 & 60.00 & {$[67.50]$} & 70.00 & 67.50 & 52.50 & 62.50 & {$[57.50]$} & 60.00 & 67.50 \\
\hline $\mathrm{SF}_{1}-\mathrm{TF}_{1}$ & 95.00 & 60.00 & {$[65.00]$} & 80.00 & 70.00 & 55.00 & {$[55.00]$} & {$[45.00]$} & 65.00 & 70.00 \\
\hline SM1-TM1 & 70.00 & 60.00 & 70.00 & 60.00 & 65.00 & 50.00 & 70.00 & 70.00 & 55.00 & 65.00 \\
\hline X-Gender & {$[50.00]$} & {$[55.00]$} & [50.00] & 60.00 & 75.00 & [40.0o] & {$[52.50]$} & {$[47.50]$} & 62.50 & 60.00 \\
\hline $\mathrm{SF}_{1}-\mathrm{TM} 1$ & [20.00] & 40.00 & 35.00 & 40.00 & 60.00 & 20.00 & 45.00 & 35.00 & 50.00 & 40.00 \\
\hline $\mathrm{SM}_{1}-\mathrm{TF}_{1}$ & 80.00 & 70.00 & {$[65.00]$} & 80.00 & 90.00 & [6o.oo] & [6o.oo] & [6o.oo] & {$[75.00]$} & 80.00 \\
\hline
\end{tabular}

S-Gender and X-Gender, respectively, denotes same-gender and cross-gender conversions. Bold indicates the system(s) with the best similarity score in each conversion category. [·] Denotes a system with a statistically significant lower score than the best score in each conversion category. Statistical inferences were performed using the two-tailed Mann-Whitney test with $\alpha<0.05$.

\section{CONCLUSION}

In this paper, we have presented a study on the use of NN-based statistical models for both spectral mapping and waveform generation in a parallel VC system. Several architectures of NN-based spectral mapping models are presented, including DNN-, DMDN-, and RNN-based architectures, i.e. by using LSTM/GRU units. Then, we have presented the use of $\mathrm{WN}$ vocoder as a state-of-the-art NNbased waveform generator (neural vocoder) for VC. The problem of quality degradation faced by the $\mathrm{WN}$ vocoder in VC owing to the oversmoothed characteristics of the estimated parameters is handled through post-conversion processing based on the direct waveform modification with spectrum differential (DiffVC) and the GV post-filter, i.e. DiffGV. Furthermore, to preserve the consistency with the DiffGV-based post-conversion method, we have proposed a modified loss function that minimizes the spectrum differential loss for the spectral modeling. The experimental results have demonstrated that: (1) the RNN-based spectral modeling, particularly the GRU-based model, achieves higher spectral mapping accuracy with a faster convergence rate and better generalization than the DNN-/DMDNbased models; (2) the RNN-based spectral modeling is also capable of generating less oversmoothed spectral trajectory than the DNN-/DMDN-based models; (3) the use of spectrum differential loss for the spectral modeling further improves the performance in same-gender conversions; and (4) the DiffGV-based post-conversion processing for the converted auxiliary speech features used in the $\mathrm{WN}$ vocoder achieves superior performance for both naturalness and speaker conversion accuracy compared to those obtained using conventional sets of converted auxiliary speech features. Future work includes the use of a GRU-based model for non-parallel VC and to directly address the mismatches of speech features for VC with the neural vocoder in a data-driven manner.

\section{FINANCIAL SUPPDRT}

This work was partly supported by the Japan Science and Technology Agency (JST), Precursory Research for Embryonic Science and Technology (PRESTO) (grant number JPMJPR1657); JST, CREST (grant number JPMJCR19A3); and Japan Society for the Promotion of Science Grantsin-Aid for Scientific Research (KAKENHI) (grant numbers JP17Ho6101 and 17Ho1763).

\section{STATEMENT OF INTEREST}

None. 


\section{REFERENCES}

1. Childers, D.B.; Yegnanarayana, B.; Wu, K.: Voice conversion: factors responsible for quality, in Proc. ICASSP, Tampa, FL, USA, March 1985, 748-751.

2. Abe, M.; Nakamura, S.; Shikano, K.; Kuwabara, H.: Voice conversion through vector quantization. J. Acoust. Soc. Jpn. (E), 11 (2) (1990), $71-76$.

3. Kain, A.; Macon, M.W.: Spectral voice conversion for text-to-speech synthesis, in Proc. ICASSP, Seattle, WA, USA, May 1998, 285-288.

4. Villavicencio, F.; Bonada, J.: Applying voice conversion to concatenative singing-voice synthesis, in Proc. INTERSPEECH, Makuhari, Japan, September 2010, 2162-2165.

5. Kobayashi, K.; Toda, T.; Nakamura, S.: Intra-gender statistical singing voice conversion with direct waveform modification using logspectral differential. Speech Commun., 99 (2018), 211-220.

6. Kain, A.B.; Hosom, J.-P.; Niu, X.; van Santen, J.P.; Fried-Oken, M.; Staehely, J.: Improving the intelligibility of dysarthric speech. Speech Commun., 49 (9) (2007), 743-759.

7. Tanaka, K.; Toda, T.; Neubig, G.; Sakti, S.; Nakamura, S.: A hybrid approach to electrolaryngeal speech enhancement based on spectral subtraction and statistical voice conversion, in Proc. INTERSPEECH, Lyon, France, September 2013, 3067-3071.

8. Doi, H.; Toda, T.; Saruwatari, H.; Shikano, K.: Alaryngeal speech enhancement based on one-to-many eigenvoice conversion. IEEE/ ACM Trans. Audio Speech Lang. Process., 22 (1) (2014), $172-183$.

9. Inanoglu, Z.; Young, S.: Data-driven emotion conversion in spoken English. Speech Commun., 51 (3) (2009), 268-283.

10. Türk, O.; Schröder, M.: Evaluation of expressive speech synthesis with voice conversion and copy resynthesis techniques. IEEE/ACM Trans. Audio Speech Lang. Process., 18 (5) (2010), 965-973.

11. Subramanya, A.; Zhang, Z.; Liu, Z.; Acero, A.: Multisensory processing for speech enhancement and magnitude-normalized spectra for speech modeling. Speech Commun., 50 (3) (2008), 228-243.

12. Toda, T.; Nakagiri, M.; Shikano, K.: Statistical voice conversion techniques for body-conducted unvoiced speech enhancement. IEEE Trans. Audio Speech Lang. Process., 20 (9) (2012), 2505-2517.

13. Tobing, P.L.; Kobayashi, K.; Toda, T.: Articulatory controllable speech modification based on statistical inversion and production mappings. IEEE/ACM Trans. Audio Speech Lang. Process., 25 (12) (2017), 2337-2350.

14. Wu, Z. et al.: : ASVspoof 2015: the first automatic speaker verification spoofing and countermeasures challenge, in Proc. INTERSPEECH, Dresden, Germany, September 2015, 2037-2041.

15. Kinnunen, T. et al.: : The ASVspoof 2017 challenge: assessing the limits of replay spoofing attack detection, in Proc. INTERSPEECH, Stockholm, Sweden, August 2017, 2-6.

16. Tokuda, K.; Kobayashi, T.; Masuko, T.; Imai, S.: Mel-generalized cepstral analysis - a unified approach to speech spectral estimation, in Proc. ICSLP, Yokohama, Japan, September 1994, 1043-1046.

17. Wu, Z.; Kinnunen, T.; Chng, E.; Li, H.: Text-independent Fo transformation with non-parallel data for voice conversion, in Proc. INTERSPEECH, Makuhari, Japan, September 2010, 1732-1735.

18. Sanchez, G.; Silen, H.; Nurminen, J.; Gabbouj, M.: Hierarchical modeling of Fo contours for voice conversion, in Proc. INTERSPEECH, Singapore, September 2014, 2318-2321.

19. Sisman, B.; Li, H.; Tan, K.C.: Transformation of prosody in voice conversion, in Proc. APSIPA, Kuala Lumpur, Malaysia, December 2017, $1537-1546$
20. Dudley, H.: Remaking speech. J. Acoust. Soc. Am., 11 (2) (1939), 169-177.

21. Kawahara, H.; Masuda-Katsuse, I.; De Cheveigné, A.: Restructuring speech representations using a pitch-adaptive time-frequency smoothing and an instantaneous-frequency-based Fo extraction: possible role of a repetitive structure in sounds. Speech Commun., 27 (1999), 187-207.

22. Toda, T.; Black, A.W.; Tokuda, K.: Voice conversion based on maximum-likelihood estimation of spectral parameter trajectory. IEEE Trans. Audio Speech Lang. Process., 15 (8) (2007), 2222-2235.

23. van den Oord, A. et al.: WaveNet: A generative model for raw audio. arXiv preprint arXiv:1609.03499, 2016.

24. Tobing, P.L.; Wu, Y.-C.; Hayashi, T.; Kobayashi, K.; Toda, T.: NU voice conversion system for the Voice Conversion Challenge 2018, in Proc. Odyssey, Les Sables d'Olonne, France, June 2018, 219-226.

25. Erro, D.; Moreno, A.; Bonafonte, A.: Voice conversion based on weighted frequency warping. IEEE Trans. Audio Speech Lang. Process., 18 (5) (2010), 922-931.

26. Godoy, E.; Rosec, O.; Chonavel, T.: Voice conversion using dynamic frequency warping with amplitude scaling, for parallel or nonparallel corpora. IEEE Trans. Audio Speech Lang. Process., 20 (4) (2012), $1313-1323$.

27. Takashima, R.; Takiguchi, T.; Ariki, Y.: Exemplar-based voice conversion using sparse representation in noisy environments. IEICE Trans. Fundam. Electron. Commun. Comput. Sci., 96 (10) (2013), 1946-1953.

28. Wu, Z.; Virtanen, T.; Chng, E.S.; Li, H.: Exemplar-based sparse representation with residual compensation for voice conversion. IEEE/ACM Trans. Audio Speech Lang. Process., 22 (10) (2014), $1506-1521$.

29. Stylianou, Y.; Cappé, O.; Moulines, E.: Continuous probabilistic transform for voice conversion. IEEE Trans. Speech Audio Process., 6 (2) (1998), 131-142.

30. Desai, S.; Black, A.W.; Yegnanarayana, B.; Prahallad, K.: Spectral mapping using artificial neural networks for voice conversion. IEEE Trans. Audio Speech Lang. Process., 18 (5) (2010), 954-964.

31. Chen, L.-H.; Ling, Z.-H.; Liu, L.-J.; Dai, L.-R.: Voice conversion using deep neural networks with layer-wise generative training. IEEE/ACM Trans. Audio Speech Lang. Process., 22 (12) (2014), 1859-1872.

32. Sun, L.; Kang, S.; Li, K.; Meng, H.: Voice conversion using deep bidirectional long short-term memory based recurrent neural networks, in Proc. ICASSP, South Brisbane, Australia, April 2015, 4869-4873.

33. Nakashika, T.; Takiguchi, T.; Minami, Y.: Non-parallel training in voice conversion using an adaptive restricted Boltzmann machine. IEEE/ACM Trans. Audio Speech Lang. Process., 24 (11) (2016), 2032-2045.

34. Hsu, C.-C.; Hwang, H.-T.; Wu, Y.-C.; Tsao, Y.; Wang, H.-M.: Voice conversion from unaligned corpora using variational autoencoding Wasserstein generative adversarial networks, in Proc. INTERSPEECH, Stockholm, Sweden, August 2017, 3364-3368.

35. Kaneko, T.; Kameoka, H.: CycleGAN-VC: non-parallel voice conversion using cycle-consistent adversarial networks, in Proc. EUSIPCO, Rome, Italy, September 2018, 2100-2104.

36. Hochreiter, S.; Schmidhuber, J.: Long short-term memory. Neural Comput., 9 (8) (1997), 1735-1780.

37. Cho, K. et al.:: Learning phrase representations using RNN encoderdecoder for statistical machine translation, arXiv preprint arXiv:1406. $1078,2014$.

38. Morise, M.; Yokomori, F.; Ozawa, K.: WORLD: a vocoder-based highquality speech synthesis system for real-time applications. IEICE Trans. Inf. Syst., 99 (7) (2016), 1877-1884. 
39. Raitio, T. et al.: : HMM-based speech synthesis utilizing glottal inverse filtering. IEEE/ACM Trans. Audio Speech Lang. Process., 19 (1) (2011), 153-165.

40. Airaksinen, M.; Juvela, L.; Bollepalli, B.; Yamagishi, J.; Alku, P.: A comparison between straight, glottal, and sinusoidal vocoding in statistical parametric speech synthesis. IEEE/ACM Trans. Audio Speech Lang. Process., 26 (9) (2018), 1658-1670.

41. Erro, D.; Sainz, I.; Navas, E.; Hernaez, I.: Harmonics plus noise model based vocoder for statistical parametric speech synthesis. IEEE J. Sel. Top. Signal Process., 8 (2) (2014), 184-194.

42. Degottex, G.; Lanchantin, P.; Gales, M.: A pulse model in log-domain for a uniform synthesizer, in Proc. 9th ISCA Speech Synthesis Workshop, Sunnyvale, CA, USA, September 2016, 230-236.

43. Morise, M.; Watanabe, Y.: Sound quality comparison among highquality vocoders by using re-synthesized speech. Acoust. Sci. Technol., 39 (3) (2018), 263-265.

44. Tamamori, A.; Hayashi, T.; Kobayashi, K.; Takeda, K.; Toda, T.: Speaker-dependent WaveNet vocoder, in Proc. INTERSPEECH, Stockholm, Sweden, August 2017, 1118-1122.

45. Hayashi, T.; Tamamori, A.; Kobayashi, K.; Takeda, K.; Toda, T.: An investigation of multi-speaker training for WaveNet vocoder, in Proc. ASRU, Okinawa, Japan, December 2017, 712-718.

46. Adiga, N.; Tsiaras, V.; Stylianou, Y.: On the use of WaveNet as a statistical vocoder, in Proc. ICASSP, Calgary, Canada, April 2018, pp. 5674-5678.

47. Kobayashi, K.; Hayashi, T.; Tamamori, A.; Toda, T.: Statistical voice conversion with WaveNet-based waveform generation, in Proc. INTERSPEECH, Stockholm, Sweden, August 2017, 1138-1142.

48. Takamichi, S.; Toda, T.; Black, A.W.; Neubig, G.; Sakti, S.; Nakamura, S.: Postfilters to modify the modulation spectrum for statistical parametric speech synthesis. IEEE/ACM Trans. Audio Speech Lang. Process., 24 (4) (2016), 755-767.

49. Shen, J. et al.: : Natural TTS synthesis by conditioning WaveNet on mel spectrogram predictions, in Proc. ICASSP, Calgary, Canada, April 2018, 4779-4783.

50. Liu, L.-J.; Ling, Z.-H.; Jiang, Y.; Zhou, M.; Dai, L.-R.: WaveNet vocoder with limited training data for voice conversion, in Proc. INTERSPEECH, Hyderabad, India, September 2018, 1983-1987.

51. Lorenzo-Trueba, J. et al:: : The voice conversion challenge 2018: promoting Development of Parallel and Nonparallel Methods, arXiv preprint arXiv:1804.04262, 2018.

52. Zen, H.; Senior, A.: Deep mixture density networks for acoustic modeling in statistical parametric speech synthesis, in Proc. ICASSP, Florence, Italy, May 2014, 3844-3848.

53. Tokuda, K.; Kobayashi, T.; Imai, S.: Speech parameter generation from HMM using dynamic features, in Proc. ICASSP, Detroit, MI, USA, May 1995, 660-663.

54. Mashimo, M.; Toda, T.; Shikano, K.; Campbell, N.: Evaluation of cross-language voice conversion based on GMM and STRAIGHT, in Proc. EUROSPEECH, Aalborg, Denmark, September 2001, 361-364.

55. Imai, S.; Sumita, K.; Furuichi, C.: Mel log spectrum approximation (MLSA) filter for speech synthesis. Electron. Commun. Jpn. I, 66 (2) 10-18, 1983.

56. Kominek, J.; Black, A.: The CMU Arctic speech databases for speech synthesis research, Language Technologies Institute, Carnegie Mellon University, 2003. [Online]. Available at http://festvox.org/cmuarctic

57. Hsu, C.-C.: PyWorldVocoder - a Python wrapper for WORLD vocoder. [Online]. Available at https://github.com/JeremyCCHsu/ Python-Wrapper-for-World-Vocoder
58. Yamamoto, R.: A python wrapper for speech signal processing toolkit (SPTK).' [Online]. Available at https://github.com/rgyg/pysptk

59. Morise, M.: CheapTrick, a spectral envelope estimator for highquality speech synthesis. Speech Commun., 67 (2015), 1-7.

6o. Morise, M.: Error evaluation of an Fo-adaptive spectral envelope estimator in robustness against the additive noise and Fo error. IEICE Trans. Inf. Syst., E98-D (7) (2015), 1405-1408.

61. Morise, M.: A high-performance fundamental frequency estimator from speech signals, in Proc. INTERSPEECH, Stockholm, Sweden, August 2017, 2321-2325.

62. Morise, M.: $\mathrm{D}_{4} \mathrm{C}$, a band-aperiodicity estimator for high-quality speech synthesis. Speech Commun., 84 (2016), 57-65.

63. T., Hayashi: Pytorch-WaveNet-Vocoder. [Online]. Available at https://github.com/kan-bayashi/PytorchWaveNetVocoder

64. Tachibana, K.; Toda, T.; Shiga, Y.; Kawai, H.: An investigation of noise shaping with perceptual weighting for WaveNet-based speech generation, in Proc. ICASSP, Calgary, Canada, April 2018, 5664-5668.

65. Kingma, D. P.; Ba, J.: Adam: A method for stochastic optimization. arXiv preprint arXiv:1412.6980, 2014.

66. Glorot, X.; Bengio, Y.: Understanding the difficulty of training deep feedforward neural networks, in Proc. AISTATS, Sardinia, Italy, May 2010, 249-256.

67. Srivastava, N.; Hinton, G.; Krizhevsky, A.; Sutskever, I.; Salakhutdinov, R.: Dropout: a simple way to prevent neural networks from overfitting. J. Mach. Learn. Res., 15 (1) (2014), 1929-1958.

68. Kobayashi, K.; Toda, T.: sprocket: open-source voice conversion software, in Proc. Odyssey, Les Sables d'Olonne, France, June 2018, 203-210.

Patrick Lumban Tobing received his B.E. degree from Bandung Institute of Technology (ITB), Indonesia, in 2014 and his M.E. degree from Nara Institute of Science and Technology (NAIST), Japan, in 2016. He received his Ph.D. degree in information science from the Graduate School of Information Science, Nagoya University, Japan, in 2020, and is currently working as a Postdoctoral Researcher. He received the Best Student Presentation Award from the Acoustical Society of Japan (ASJ). He is a member of IEEE, ISCA, and ASJ.

Yi-Chiao Wu received his B.S and M.S degrees in engineering from the School of Communication Engineering of National Chiao Tung University in 2009 and 2011, respectively. He worked at Realtek, ASUS, and Academia Sinica for 5 years. Currently, he is pursuing his Ph.D. degree at the Graduate School of Informatics, Nagoya University. His research topics focus on speech generation applications based on machine learning methods, such as voice conversion and speech enhancement.

Tomoki Hayashi received his B.E. degree in engineering and his M.E. and Ph.D. degrees in information science from Nagoya University, Nagoya, Japan, in 2013, 2015, and 2019, respectively. He is currently working as a Postdoctoral Researcher at Nagoya University. His research interests include statistical speech and audio signal processing. He received the Acoustical Society of Japan (ASJ) 2014 Student Presentation Award. He is a member of IEEE and ASJ.

Kazuhiro Kobayashi received his B.E. degree from the Department of Electrical and Electronic Engineering, Faculty of Engineering Science, Kansai University, Japan, in 2012, and 
his M.E. and Ph.D. degrees from Nara Institute of Science and Technology (NAIST), Japan, in 2014 and 2017, respectively. He is currently working as a Postdoctoral Researcher at the Graduate School of Information Science, Nagoya University, Japan. He has received a few awards including the Best Presentation Award from the Acoustical Society of Japan (ASJ). He is a member of IEEE, ISCA, and ASJ.

Tomoki Toda received his B.E. degree from Nagoya University, Japan, in 1999 and his M.E. and D.E. degrees from Nara Institute of Science and Technology (NAIST), Japan, in
2001 and 2003, respectively. He was a Research Fellow of the Japan Society for the Promotion of Science from 2003 to 2005. He was then an Assistant Professor (2005-2011) and an Associate Professor (2011-2015) at NAIST. Since 2015, he has been a Professor in the Information Technology Center at Nagoya University. His research interests include statistical approaches to speech and audio processing. He has received more than 10 paper/achievement awards including the IEEE SPS 2009 Young Author Best Paper Award and the 2013 EURASIP-ISCA Best Paper Award (Speech Communication Journal). 